\title{
BREVE HISTORIA DEL HUMO DE LEÑA Y SUS IMPLICACIONES PARA CHILE*1
}

\author{
Kirk R. Smith y Ajay Pillarisetti \\ University of California, Berkeley
}

Resumen: Cuando viven cerca de bosques, los seres humanos siempre han usado la madera como combustible. Hoy en día, el empleo de la madera en todo el mundo depende en gran medida de su disponibilidad y tiene poco que ver con el consumo per cápita en todos los ingresos nacionales, aunque

KIRK R. SMIth. Ph.D. en Biomédica y Salud Ambiental de la Universidad de California, Berkeley. Profesor de Salud Ambiental de la misma universidad, además de fundador y director de su Programa Global de Salud y Ambiente. Miembro de la U.S. National Academy of Sciences, 1997, e investigador de la International Academy of Indoor Air Sciences, 1997. En abril de 2012 fue galardonado con el Premio Tyler de Medio Ambiente. Dirección electrónica: krksmith@ berkeley.edu.

Ajay Pillarisetti. Estudiante de doctorado en ciencias de la salud del medio ambiente en la Universidad de California, Berkeley. Ha trabajado en proyectos de salud y del medio ambiente en Guatemala, Grenada, India y Nepal. Dirección electrónica: ajaipillarisetti@gmail.com.

* Conferencia presentada en el seminario "Uso de Leña: Implicaciones en Salud y Clima" realizado en el Centro de Estudios Públicos, Santiago, Chile, el 21 de junio de 2011.

Traducción al castellano por Alberto Ide para Estudios Públicos. Versión en inglés en la edición en línea de Estudios Públicos (www.cepchile.cl).

1 Agradecemos a Guillermo González Caballero y a Cristóbal de la Maza Guzmán por sus aclaraciones técnicas y por revisar los borradores de este manuscrito.

Estudios Públicos, 126 (otoño 2012). 
representa un pequeño porcentaje del consumo total de energía en países más ricos a medida que otras fuentes de energía adquieren predominancia. En los países pobres, la madera se utiliza en forma intensiva para cocinar, pero más que nada para calefaccionar espacios interiores, y como combustible en pequeñas industrias conforme aumentan los ingresos. Sin embargo, en los sencillos artefactos domésticos que se emplean ya sea para cocinar o para calefaccionar el hogar, la combustión de la leña genera un alto grado de contaminación ambiental que, en forma de partículas finas o de muchos otros componentes tóxicos, resulta nociva para la salud. En Chile y en otros países de zonas templadas de ambos hemisferios donde la madera se usa para calefaccionar un alto porcentaje de los hogares durante el invierno, la contaminación externa puede alcanzar niveles que producen graves impactos en la salud, de lo cual existe una creciente conciencia. Por ejemplo, en algunas ciudades chilenas, sobre todo en aquellas situadas en el sur del país, las cardiopatías pueden aumentar hasta en un $50 \%$ como consecuencia de la contaminación del aire. Por tanto, es de esperar que la introducción en Chile de combustibles de combustión más limpia, como el gas, o de estufas a leña con una alta eficiencia de combustión, redunde en importantes beneficios para la salud dentro de algunos años, cuando se logre implementar programas destinados a masificar su uso.

Palabras clave: contaminación ambiental intradomiciliaria, calefacción de espacios interiores, dendrocombustibles, MP2,5, contaminación ambiental por material particulado, cardiopatías.

\section{A SHORT HISTORY OF WOODSMOKE AND IMPLICATIONS FOR CHILE}

Abstract: When living near forests, humans have always used wood fuel. Today its use across the world is a function mainly of wood availability with little relation in the amount used per capita across national incomes, although it constitutes a smaller fraction of total energy in wealthier countries as other energy sources become dominant. In poor countries, much of the use is for cooking while more is used for space heating and small industries as incomes rise. In simple household stoves, whether for cooking or heating, however, wood fuel produces significant health-damaging air pollution 
in the form of small particles as well as many other toxic components. In Chile and other countries in temperate areas of both hemispheres where wood is used for heating for a large portion of households in the winter, the levels of outdoor pollution can reach levels that are increasingly understood to produce significant health impacts. Heart disease, for example, may be increased as much as 50\% by this pollution in some Chilean cities, particularly in the south. In Chile, cleaner burning fuels such as gas or highcombustion-efficiency woodstoves can thus be expected to have major benefits for health within a few years after successful programs to disseminate them widely.

Keywords: Household air pollution, space heating, wood fuel, PM2.5, particle air pollution, heart disease.

\section{Introducción}

E

n su libro World Fire ("El fuego en el mundo"), el historiador Stephen Pyne señala que a lo largo de la historia, dondequiera que los seres humanos han vivido en las cercanías de bosques, ha aumentado la tasa de combustión de madera (Pyne, 1995). Lo anterior ocurre en parte por incendios forestales accidentales de origen humano, el manejo del fuego en predios forestales y el uso humano de la madera como combustible. Hasta cierto punto existe un trade off: mientras menos leña se extraiga de los bosques para combustible, mayor será la magnitud del próximo incendio. Algunos bosques pueden descomponerse, pero la mayoría se quemará en algún momento. Los incendios forestales son un fenómeno natural y necesario para mantener la salud de los ecosistemas silvícolas (Pyne, 2001). Puede afirmarse, asimismo, que el uso humano de los dendrocombustibles es una actividad, si no precisamente natural, por lo menos inevitable y necesaria para mantener la salud de las sociedades humanas. Es más, el acontecimiento que muchos arqueólogos utilizan para definir el momento en que las condiciones de la humanidad cambiaron de prehumanas a humanas fue el hecho de aprender a controlar el fuego (Lévi-Strauss, 1969). Cuesta creer que la fecha aproximada en que la cocina a leña se transformó en un componente habitual de las habitaciones humanas - hace unos 350.000 años - haya sido determinada solo recientemente (Roebroeks y Villa, 2011). De modo que hemos estado usando la cocina a leña desde hace mucho tiempo. 


\section{Uso de la madera y desarrollo}

Hoy en día, el uso de la madera y de otros biocombustibles en los hogares es considerado algunas veces un indicador de pobreza: las poblaciones de escasos recursos sin acceso a combustibles modernos deben recurrir a la extracción de biocombustibles para satisfacer sus necesidades domésticas de cocinar alimentos y calefaccionarse. Lo cierto es que estas actividades que se iniciaron hace un tercio de millón de años siguen realizándose diariamente en más del $40 \%$ de los hogares del mundo. Esta afirmación se basa, al parecer, en el hecho de que el porcentaje de uso de la energía nacional atribuible a la madera y a otros biocombustibles tiende a disminuir con el desarrollo a medida que otras fuentes de energía adquieren mayor relevancia, como se indica en la Figura $\mathrm{N}^{\circ} 1$.

FIGURA N ${ }^{\circ}$ 1: $\quad$ CAMBIOS EN LA PROPORCIÓN DE ENERGÍA TOTAL POR TIPOS DE COMBUSTIBLE / ENERGÍA DESDE 1850

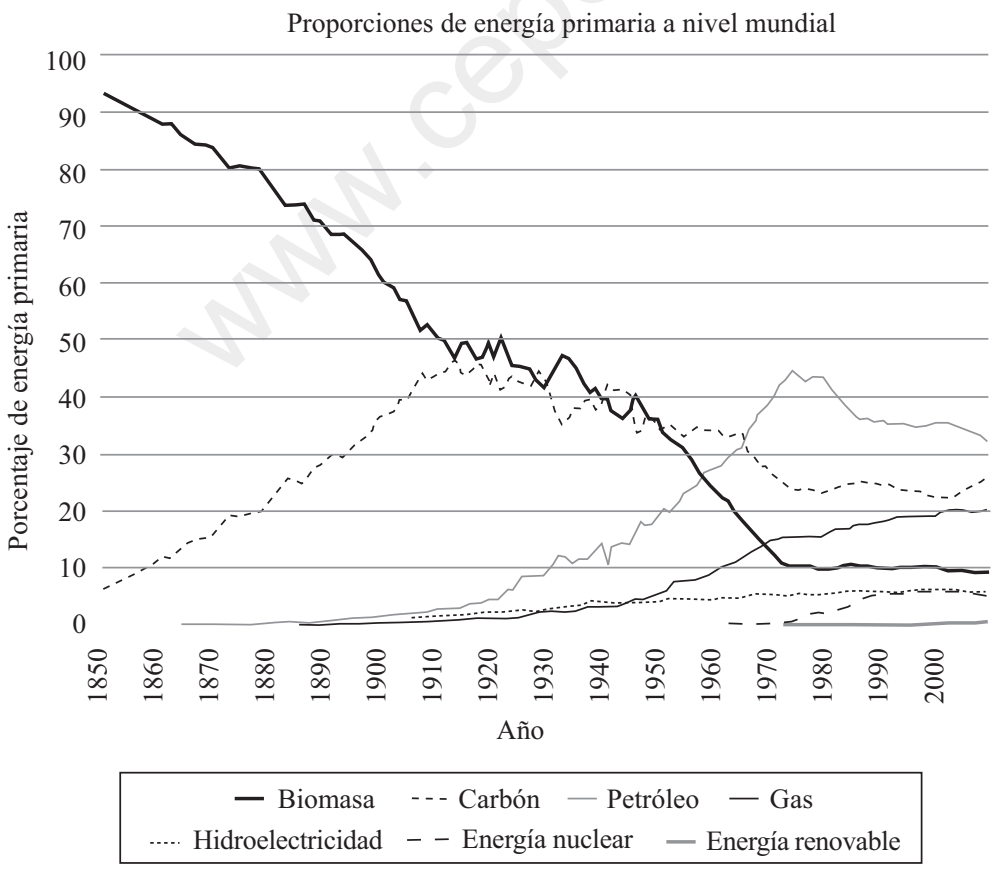

Nota: Según esta medición, la biomasa se ha vuelto mucho menos importante a nivel mundial con el paso del tiempo.

Fuente: GEA (2012). 
Sin embargo, cuando el uso del biocombustible se formula como una fracción del consumo total de energía, pasa inadvertido un aspecto más pertinente para este análisis, a saber, que el consumo absoluto de leña per cápita tiene escasa relación con el desarrollo. Como se muestra en la Figura $\mathrm{N}^{\circ} 2$, si bien existe un alto grado de variación, no se aprecia una tendencia evidente a medida que aumentan los ingresos.

La variación observada parece explicarse simplemente por la disponibilidad. Singapur y Finlandia son ambos países ricos, pero el primero casi no tiene bosques y su consumo de dendrocombustibles es prácticamente nulo, mientras que en el segundo se observa una enorme superficie boscosa y un alto consumo de leña. Por cierto que las maneras de utilizar la leña dependen del nivel de ingresos; así por ejemplo,

FIGURA N ${ }^{\circ}$ 2: $\quad$ ENERGÍA DE BIOMASA PER CÁPITA POR INGRESO NACIONAL
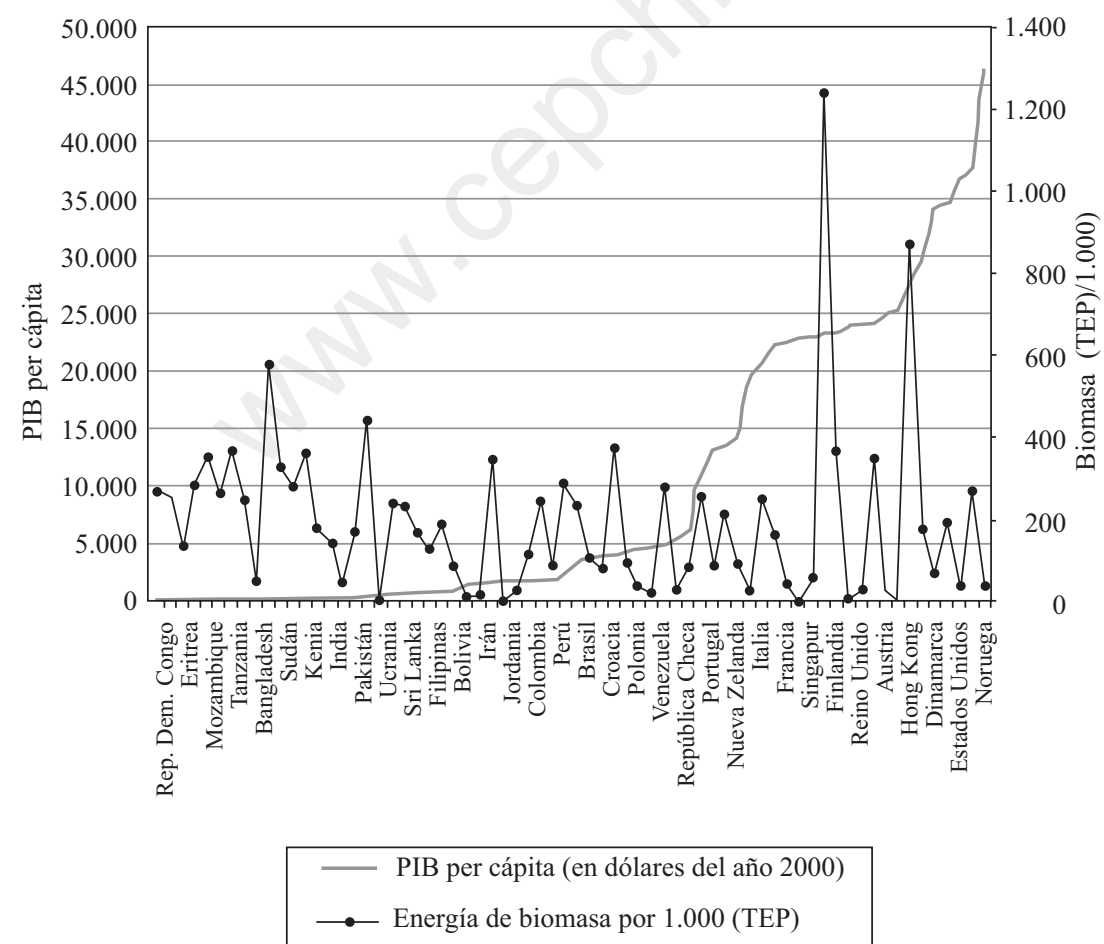

Nota: Nótese que aunque existe un alto grado de variación, no se observa una tendencia clara.

Fuente: World Resources Institute (2007). 
el porcentaje de hogares donde se cocina con biomasa disminuye considerablemente mientras mayor es el ingreso, pero otros usos de la leña pueden mantenerse o incluso incrementarse, incluidas las chimeneas, las calderas o las pequeñas centrales eléctricas, etc. Con todo, cuando la madera está disponible siempre existe la posibilidad de que combustione en algún momento, incluso en el próximo incendio forestal. Existe, asimismo, una situación paralela asociada a los residuos agrícolas que son recolectados y usados como combustible para satisfacer necesidades domésticas en los países pobres, o que suelen ser quemados a campo abierto, incluso en los países más ricos. Tal como sucede con la leña, la cantidad total de desechos quemados dependerá de la disponibilidad, en este caso de la extensión y los tipos de cultivos existentes a nivel local.

En consecuencia, tal como se observa en la Figura $\mathrm{N}^{\circ} 3$, la humanidad ha consumido casi la misma cantidad absoluta de biocombustible a lo largo de cientos de años y, si corregimos por la variable demográfica, probablemente durante mucho más tiempo. Cuando hay biocombustible disponible, éste se utiliza.

\section{Humo de leña}

En décadas recientes, en parte debido a la sofisticación de las ciencias de la salud (la epidemiología y la toxicología) y en parte al cambio en las expectativas respecto de la protección sanitaria, el material particulado producido por la combustión ha llegado a ser considerado un grave riesgo para la salud en casi todos los países. Por ejemplo, en la nueva Global Energy Assessment se señala que unos cinco millones de personas mueren prematuramente cada año como consecuencia de las emisiones de los biocombustibles, incluso sin considerar el tabaquismo activo y pasivo, cuyos efectos sobre la salud también son atribuibles a sustancias contaminantes producidas por la combustión de biomasa. Estos efectos derivan de los riesgos asociados a la contaminación del aire doméstico producida por el uso de biomasa y carbón, y a la contaminación atmosférica exterior por material particulado, siendo ambas atribuibles principalmente a una deficiente combustión.

Tal como se describe más adelante, tal vez resulte sorprendente que, desde una amplia perspectiva en el área de la salud, existan muy pocas referencias que nos permitan distinguir las partículas entre las 
FIGURA N ${ }^{\circ}$ 3: $\quad$ USO DE ENERGÍA POR EL HOMBRE DESDE 1850 SEGÚN PRINCIPALES TIPOS DE COMBUSTIBLE/ENERGÍA

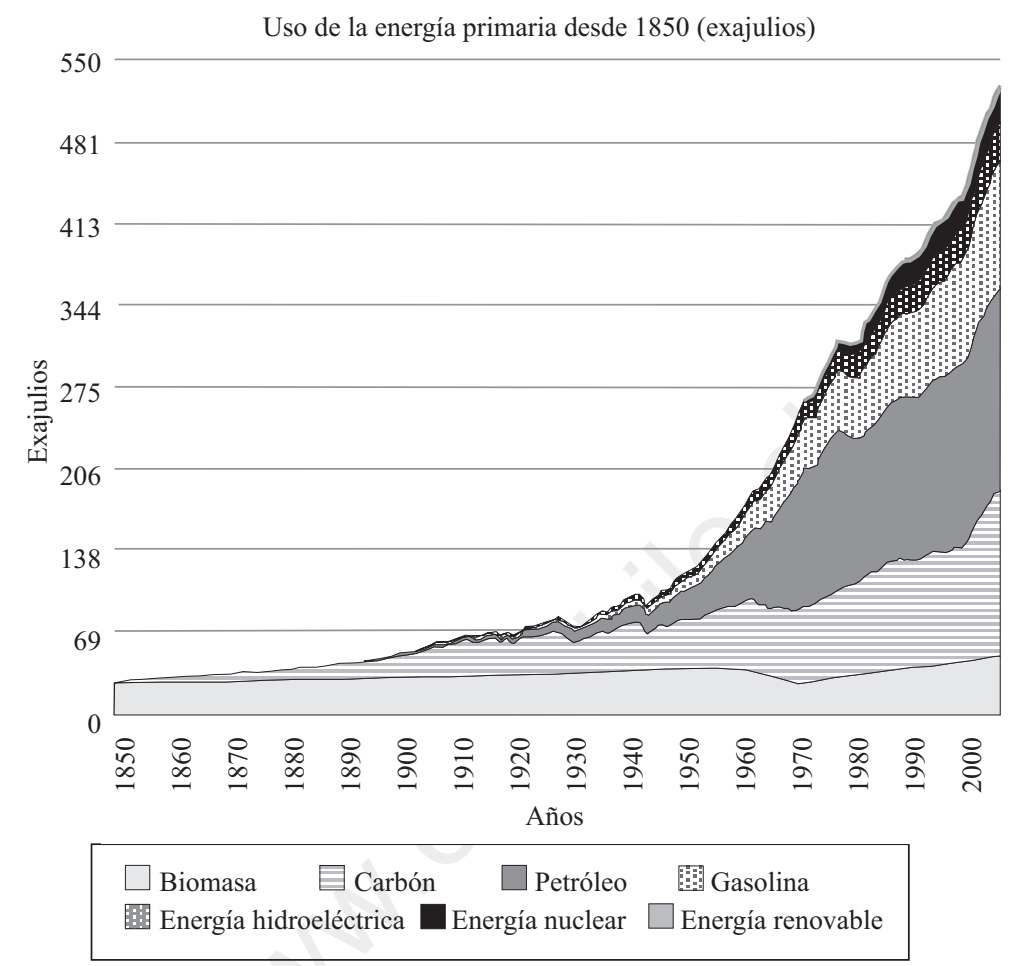

Nota: Nótese que no existe una tendencia para la cantidad total de biocombustible usado durante este período.

Fuente: GEA (2012).

distintas fuentes, ya sean combustibles fósiles, leña o tabaco, por ejemplo. A menos que vayan acompañadas por determinados contaminantes tóxicos, las partículas de combustión son todas igualmente perniciosas para la salud humana, por cierto que en función de la dosis recibida.

La aplicación de quemas para limpiar la tierra (desbroce) ha producido un enorme impacto en el medio ambiente mundial, incluso cuando la población humana era relativamente escasa (Rambo, 1982). Así pues, cuando se desarrolló la cocina a leña la población de la Tierra probablemente rondaba el millón de habitantes, por lo que el impacto de la combustión de la madera en el hogar era relativamente pequeño incluso a nivel local, aunque tal vez acabara generando riesgos para la 
salud de los usuarios. Sin embargo, en la actualidad, con siete mil millones de habitantes, el impacto de la combustión de biomasa en varios cientos de millones de hogares es realmente enorme. Por ejemplo, se calcula que la contaminación atmosférica derivada del uso de biomasa para cocinar produce la muerte de 2,5 millones de personas anualmente en países pobres (IIASA, 2012). Debido a que el humo de los biocombustibles contiene una gran cantidad de contaminantes que alteran el clima, se estima que el consumo doméstico de biomasa será uno de los principales sectores que influirán en el cambio climático, compitiendo con el transporte terrestre, la producción ganadera, las centrales eléctricas, etc. (Unger y otros, 2010).

El humo de biomasa es también un importante factor que contribuye a la contaminación ambiental en muchos lugares del mundo. Las quemas intencionales para fines de desbroce siguen siendo una práctica habitual en partes de África, Indonesia y Brasil, causando un enorme impacto en la calidad del aire. En otras regiones se observa un alto nivel de contaminación atribuible a incendios forestales ocasionales o a la quema estacional de residuos agrícolas. En algunas poblaciones pobres, el uso diario de biocombustibles para cocinar es una importante fuente de contaminación; por ejemplo, en India y en China el uso doméstico del combustible es el responsable de alrededor de un 50\% y un 30\%, respectivamente, de todas las emisiones externas de material particulado (Chafe y Smith, 2010). Con todo, en gran parte del mundo la contaminación ambiental está poderosamente influida por el uso de leña para la calefacción de espacios interiores, de modo que la situación empeora en los meses de invierno.

Aun cuando no existen inventarios sistemáticos de emisiones a nivel mundial en los que se considere la combustión de leña para calefaccionar espacios interiores, no cabe duda de que ella es responsable de más de la mitad de las emisiones externas de material particulado en muchos países, por ejemplo en Canadá, Finlandia, Noruega, Dinamarca, Nueva Zelanda y Chile. Durante el invierno su predominio se extiende incluso a más regiones del mundo, entre ellas gran parte de los Estados Unidos; Australia; Europa Central, Oriental y Occidental, y Rusia. Si se incluyera la calefacción con carbón, la combustión doméstica sería responsable del grueso de todas las emisiones de material particulado en Mongolia y, si no de la mayoría, de un alto porcentaje de las emisiones en la templada China durante el invierno. Por ejemplo, el uso doméstico 
del carbón para calefaccionar espacios internos en Ulán Bator, Mongolia, genera uno de los peores fenómenos de contaminación atmosférica externa en el mundo durante la temporada fría.

Las conclusiones anteriores resultan alarmantes, ya que en todos los países mencionados existe un alto nivel de quema de combustibles fósiles en los sectores industrial, de transportes y comercial, con la consiguiente emisión de material particulado. En la mayoría de los casos, los dendrocombustibles representan una proporción relativamente pequeña del consumo total de energía, pero teniendo en cuenta los altos índices de emisiones, generalmente incontroladas, por unidad de energía, la combustión de leña en los hogares produce un impacto desproporcionado en la calidad del aire.

\section{¿Cuál es el problema?}

Como ya se señaló, la humanidad ha estado expuesta al humo de leña desde los comienzos de su historia. En vista de lo anterior, hay quienes a veces sostienen que, como se trata de un proceso "natural", tiene que ser benigno, afirmación que a todas luces constituye un despropósito. Hay muchos elementos naturales que resultan bastante perjudiciales, desde el veneno de serpiente hasta la toxina botulínica, el arsénico, el virus de la malaria, por no mencionar fenómenos como los tifones, las inundaciones y las sequías. Lo cierto es que el grueso de la humanidad se ha pasado la mayor parte de su historia protegiéndose contra las amenazas naturales.

Puesto que el humo es una mezcla de sustancias químicas, lo lógico sería preguntarse si se ha demostrado que alguna de ellas constituye un riesgo para la salud. Desgraciadamente, la respuesta es sí. Se han identificado miles de sustancias químicas en el humo de leña, de las cuales cientos son reconocidamente peligrosas. Entre ellas hay muchas que han sido catalogadas como productos químicos de uso industrial, por ejemplo el benceno, el formaldehído, los hidrocarburos poliaromáticos y la dioxina. Asimismo, los niveles encontrados en casos de masivas emisiones de humo de leña - el cual contiene esas sustancias químicas - compiten con aquellos detectados en fábricas y otros modernos ambientes contaminados donde se han registrado efectos en la salud (Naeher y otros, 2007; IARC, 2010). 
El humo de biomasa mejor estudiado es el que se desprende de la combustión del tabaco, que contiene los mismos componentes del humo de leña en proporciones relativas algo distintas, a los que se suman otras sustancias derivadas de la nicotina y de los aditivos incorporados durante la fabricación de los cigarrillos. Con todo, no se considera que ellos sean el origen de la mayoría de los efectos en la salud, sino más bien el producto de una combustión incompleta.

Aun cuando son miles las sustancias químicas presentes en los humos de combustión, incluidos los de la leña y el tabaco, no es posible medirlos de manera regular debido a obstáculos asociados al costo y a aspectos técnicos. Y tampoco resulta necesario, porque bastan unos cuantos contaminantes de referencia para establecer un vínculo con la mayoría de los tipos de riesgo para la salud. Por ejemplo, en los cigarrillos es el "alquitrán", término usado para referirse a lo que los especialistas en contaminación atmosférica denominan partículas respirables, aquellas que son lo suficientemente pequeñas para penetrar en los tejidos pulmonares profundos (y cuya medición más precisa es MP2,5: partículas con un diámetro inferior a 2,5 $\mu \mathrm{m}$ ). Para la contaminación atmosférica, MP2,5 es el más preciso de todos los indicadores del riesgo para la salud de los contaminantes derivados de la combustión, ya se trate de la contaminación atmosférica exterior o en ambientes cerrados.

\section{Las últimas investigaciones sobre efectos en la salud}

Hasta no hace mucho, las investigaciones sobre los efectos de la contaminación atmosférica en la salud se realizaban independientemente de aquellas centradas en el humo del tabaco. En los hechos, aun cuando existe, sin duda, una estrecha relación entre ambas, la diferencia radica en los altos niveles de exposición al humo del cigarrillo en comparación con la mayoría de las situaciones de contaminación del aire. Sin embargo, últimamente se están publicando cada vez más trabajos en que se cuantifica directamente la relación entre la contaminación atmosférica por partículas en niveles de exposición que fluctúan entre baja intensidad (contaminación atmosférica ambiental) y extremadamente alta intensidad (tabaquismo activo) (Pope y otros, 2011; Pope y otros, 2009). Lo anterior revela no solo un progresivo aumento en el riesgo al pasar del actual al siguiente tipo de exposición 
a la contaminación por partículas, sino además algunas notables implicaciones en cuanto a los beneficios de las medidas de control de la contaminación atmosférica en cada uno de los niveles de exposición (Smith y Peel, 2010).

En la Figura $\mathrm{N}^{\circ} 4$ se grafica esta relación para varias enfermedades importantes relacionadas con la exposición a la contaminación atmosférica, que tienen que ver con cardiopatías, tal vez el principal

FIGURA N ${ }^{\circ} 4: \quad$ RELACIÓN INTEGRADA EXPOSICIÓN-RESPUESTA PARA LA CONTAMINACIÓN POR MATERIAL PARTICULADO (MP2,5)

(Proveniente de tres principales categorías de fuentes de combustión: contaminación del aire ambiente, humo de tabaco inhalado en forma pasiva, y tabaquismo activo)

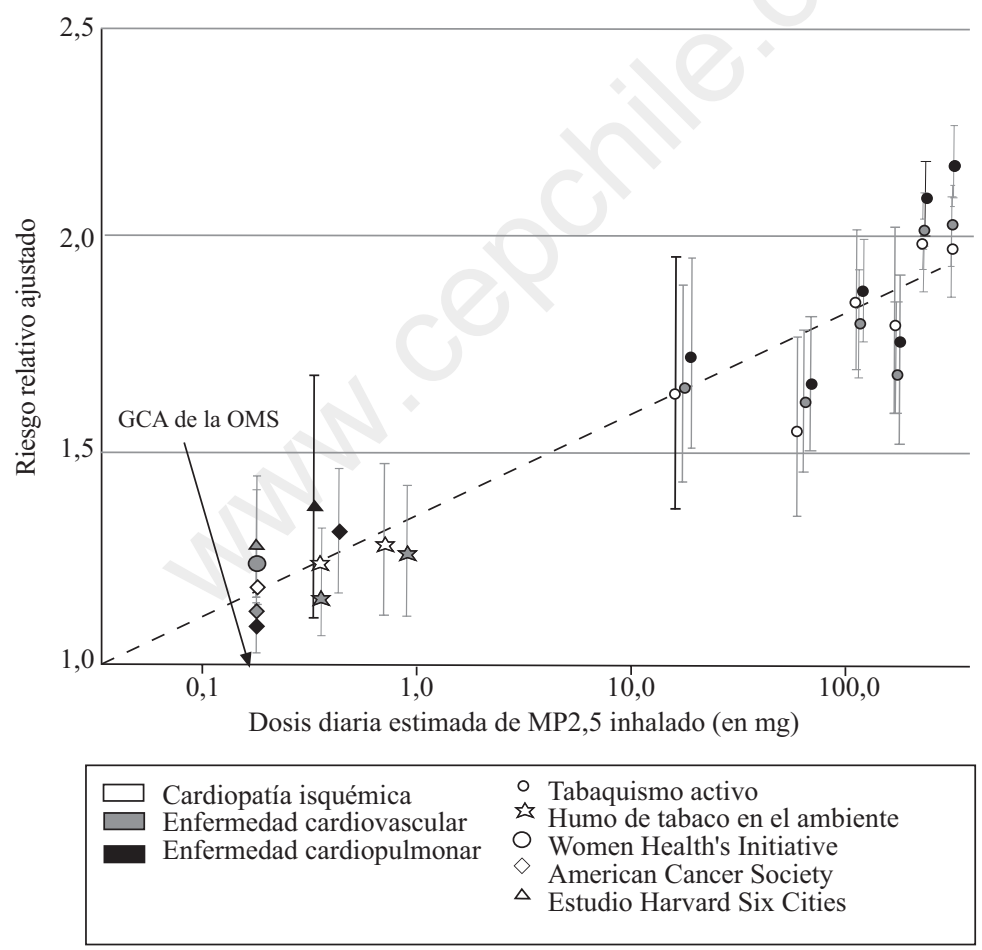

Nota: La dosis se expresa en material particulado de combustión inhalado diariamente, y el riesgo se expresa en múltiplos de enfermedades de fondo. Así pues, un riesgo relativo de 1,5 significa que las personas sometidas a este nivel de exposición sufren $1,5 \mathrm{x}(50 \%)$ más enfermedades que si no estuvieran expuestas. Esta cifra, que es una versión simplificada de la que aparece en Pope y otros (2009), proviene de Smith y Peel (2010).

Fuente: Reproducido con la debida autorización de Environmental Health Perspectives. 
efecto en la salud. Nótese que si bien la dosis de partículas inhaladas diariamente varía por un factor de 1.000 entre los fumadores compulsivos y las personas que viven en ciudades contaminadas, el riesgo de enfermedades coronarias aumenta de manera sostenida.

Como la Figura $\mathrm{N}^{\circ} 4$ incluye una escala logarítmica para exposición/dosis con unidades poco comunes $(\mathrm{mg} / \mathrm{m} 3$ por día), es difícil de interpretar. La Figura $\mathrm{N}^{\circ} 5$ expresa los resultados en una cifra con unidades más habituales, promedio anual en $\mu \mathrm{g} / \mathrm{m} 3$, que son las que suelen utilizarse en las mediciones y en las normas sobre contaminación atmosférica exterior. Se concentra en el más importante de los tipos de enfermedad coronaria: la cardiopatía isquémica (CI).

Las cifras de los estudios disponibles sobre cardiopatías asociadas a la contaminación atmosférica exterior fluctúan hasta un máximo de alrededor de $30 \mu \mathrm{g} / \mathrm{m} 3$, y las de los estudios sobre exposición pasiva

FIGURA N ${ }^{\circ}$ 5: $\quad$ RIESGO DE ENFERMEDAD CORONARIA VERSUS EXPOSICIÓN PROMEDIO ANUAL A MP2,5

Riesgos de cardiopatía isquémica derivados de la inhalación de partículas de combustión. Concentración promedio anual de MP2,5 en $\mu \mathrm{g} / \mathrm{m} 3$

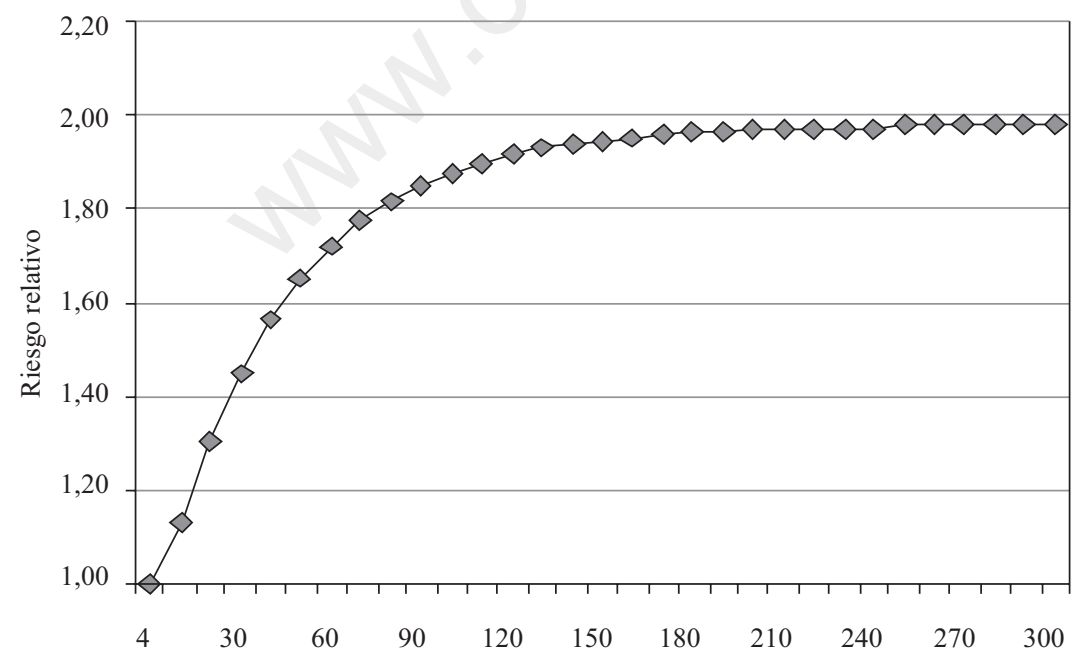

Nota: La relación integrada exposición-respuesta incorpora los resultados de estudios sobre contaminación atmosférica externa, el tabaquismo pasivo y el tabaquismo activo.

Fuente: Se han tomado como base los datos que aparecen en Pope y otros (2011). 
al humo del cigarrillo fluctúan hasta un máximo que bordea los $80 \mu \mathrm{g} /$ m3. No obstante, en este gráfico los fumadores se sitúan en el extremo derecho (10.000 o más $\mu \mathrm{g} / \mathrm{m} 3$ ).

Una característica sorprendente de la relación presentada es que el riesgo añadido por unidad de exposición adicional es bastante diferente en distintos niveles de exposición. Por ejemplo, después de 150 $\mu \mathrm{g} / \mathrm{m} 3$ el riesgo solo aumenta levemente durante el ascenso hasta los niveles experimentados por los fumadores activos. Este fenómeno se presenta en las publicaciones sobre el tabaco: los fumadores compulsivos y moderados corren prácticamente el mismo riesgo. Lo anterior tiene implicaciones potencialmente profundas para las políticas (Smith y Peel, 2010), tema que será analizado teniendo en cuenta el contexto chileno.

\section{Lecciones para Chile}

En comparación con el resto del mundo, el Hemisferio Occidental generalmente no se ve afectado por altos niveles de contaminación atmosférica doméstica o exterior (Brauer et al., 2011). Sin embargo, Chile constituye una excepción en cuanto a la contaminación externa debido al uso de leña para calefacción.

Aun cuando afecta a cualquier lugar del país donde se emplee la calefacción en espacios cerrados, este problema alcanza su máxima gravedad en las ciudades del sur emplazadas en valles y próximas a zonas boscosas, donde las temporadas frías son más largas y la circulación del aire es escasa durante el invierno. La Figura $\mathrm{N}^{\circ} 6$ muestra los niveles de MP2,5 medidos a lo largo de un año en una serie de ciudades chilenas, con la concentración mínima aproximada en invierno y cifras correspondientes a las peores dos semanas del año. Nótese que los niveles promedio de las ciudades situadas al sur son superiores a los de prácticamente cualquier zona importante del Hemisferio Occidental o de Europa, aunque no tan altos como en Asia o en África. Ello se debe al uso de estufas a leña en los hogares.

Los máximos niveles de contaminación alcanzados durante algunos días del año generan condiciones perjudiciales para todas las personas y probablemente amenazan la salud de quienes ya sufren enfermedades respiratorias. No obstante, los efectos en la salud más fáciles de percibir tienen que ver con los niveles promedio anuales. ¿Qué 


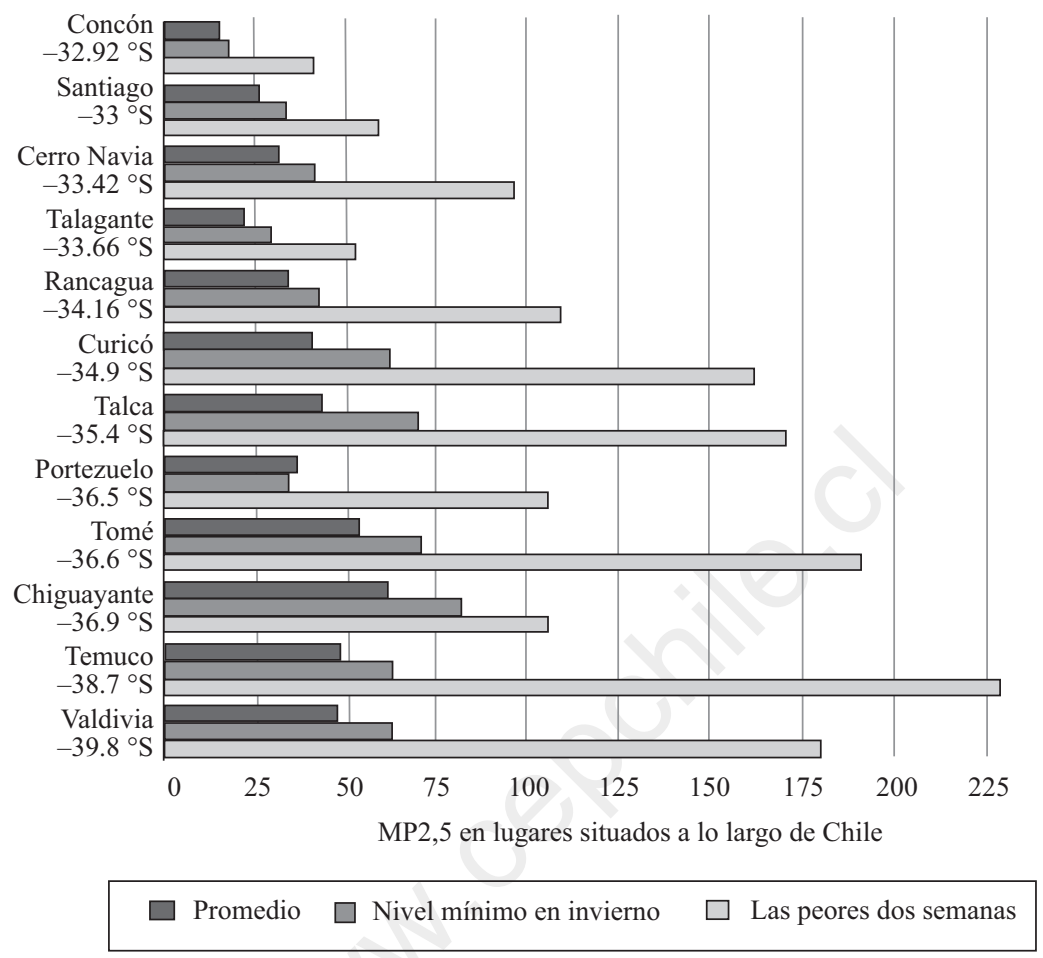

Nota: Gran parte de esta contaminación en invierno se origina en la combustión de madera en los hogares para calefacción, particularmente en las ciudades con los índices de contaminación más altos, todas las cuales se encuentran en el sur. Nótense, asimismo, los altos niveles alcanzados durante las dos peores semanas del año, fenómeno que ocurre durante la época de calefacción invernal, cuando las condiciones meteorológicas suelen impedir la dispersión de la contaminación.

Fuente: Ministerio del Medio Ambiente, Chile: SINC (Sistema de Información Nacional de Calidad del Aire) 2011.

efectos cabría prever para los habitantes de esas numerosas ciudades del sur con promedios anuales que, según las más recientes evidencias sanitarias, rondan los $50 \mu \mathrm{g} / \mathrm{m} 3$ ?

En la Figura $\mathrm{N}^{\circ} 5$ puede apreciarse que $50 \mu \mathrm{g} / \mathrm{m} 3$ corresponde a un riesgo relativo de cardiopatía de aproximadamente 1,5 , comparado con el hecho de vivir en una ciudad no contaminada con un índice de 10 $\mu \mathrm{g} / \mathrm{m} 3$, cifra recomendada por las guías de calidad del aire de la OMS (OMS, 2006). Ello implica que los habitantes de esas ciudades sufren 
un $50 \%$ más de enfermedades coronarias que la población de urbes con aire limpio, o a la inversa, que el 33\% de las cardiopatías en las ciudades del sur podrían evitarse si se lograra controlar los niveles de contaminación.

Se trata de un enorme impacto, pero la buena noticia es que los $50 \mu \mathrm{g} / \mathrm{m} 3$ se encuentran en la sección más elevada de la curva, lo que significa que las mejorías en materia de salud comenzarían a advertirse inmediatamente después de la primera medida de reducción de la contaminación. Una situación muy distinta es la que se observa, por ejemplo, en los hogares de aldeas de la India donde se queman combustibles sólidos para cocinar, cuyos niveles de exposición llegan a cientos de $\mu \mathrm{g} / \mathrm{m} 3$. En este caso, como se aprecia en la Figura $\mathrm{N}^{\circ} 5$, se requieren reducciones bastante considerables de los niveles de contaminación para observar las primeras mejorías en materia de salud.

\section{Síntesis}

Lo más perjudicial que podemos hacer es introducir un objeto en combustión en nuestra boca, que es lo que hacen millones de personas al fumar. El segundo acto más nocivo que podemos cometer es quemar una gran cantidad de sustancias al interior del hogar para cocinar a fuego abierto. El tercero, sin embargo, es algo que se observa en muchas ciudades chilenas donde para calefaccionar espacios cerrados durante el invierno se usan estufas sin mejoras en el sistema de combustión. El nivel de contaminación producido por estas estufas, presentes en la gran mayoría de los hogares, es tan alto que no se puede dispersar adecuadamente y se acumula hasta generar índices extremos de contaminación externa; además, influye en los promedios anuales con un considerable impacto en la salud.

En consecuencia, es preciso adoptar medidas para promover el uso de combustibles más limpios (como el gas), una gestión más eficiente de la energía doméstica mediante la aislación y otro tipo de medidas, y el uso de estufas a leña con un nivel de emisión significativamente inferior al de las que comúnmente se emplean en la actualidad. Cuando estas medidas comiencen a traducirse en una reducción de las emisiones y concentraciones anuales, los beneficios para la salud comenzarán a notarse de manera inmediata. 


\section{REFERENCIAS}

Brauer, M., M. Amann, R. Burnett y otros (2011). "Exposure Assessment for Estimation of the Global Burden of Disease Attributable to Outdoor Air Pollution". Environmental Science and Technology, DOI: 10.1021/ es2025752.

Chafe, Z. y K. R. Smith (2010). "Estimating the Proportion of Fine Particulate Ambient Air Pollution (PM2.5) Attributable to Household Fuel Use for Cooking in Asia”. Tesis de magíster, Universidad de California, Berkeley.

GEA (2012). Global Energy Assessment: Toward a Sustainable Future. IIASA, Viena, y Cambridge University Press.

IARC (2010). "Household Use of Solid Fuels and High-temperature Frying. (IARC Monograph 95 on the Evaluation of Carcinogenic Risks to Humans). International Agency for Research on Cancer: Lyon, Francia.

IIASA (International Institute for Applied Systems Analysis) (2012). Global Energy Assessment. Laxenburg, Austria: International Institute for Applied Systems Analysis.

Lévi-Strauss, C. (1969). The Raw and the Cooked. Nueva York: Harper \& Row, primera edición estadounidense.

Ministerio de Medio Ambiente, Gobierno de Chile: SINC (Sistema de Información Nacional de Calidad del Aire), 2011. Http://sinca.mma.gob.cl.

Naeher, L. P., M. Brauer, M. Lipsett y otros (2007). "Woodsmoke Health Effects: A Review". Journal of Inhalation Toxicology 19 (1): 67-106.

OMS (2006). Guías de Calidad del Aire de la OMS Relativas al Material Particulado, el Ozono, el Dióxido de Nitrógeno y el Dióxido de Azufre. Organización Mundial de la Salud: Ginebra.

Pope, C. A., 3rd, R. T. Burnett, D. Krewski y otros (2009). "Cardiovascular Mortality and Exposure to Airborne Fine Particulate Matter and Cigarette Smoke: Shape of the Exposure-Response Relationship". Circulation 120 (11): 941-948.

Pope, C. A. 3rd, R. T. Burnett, M. C. Turner y otros (2011). "Lung Cancer and Cardiovascular Disease Mortality Associated with Ambient Air Pollution and Cigarette Smoke: Shape of the Exposure-Response Relationships". Environmental Health Perspectives 119 (11): 16161621.

Pyne, S. J. (1995). World Fire: The Culture of Fire on Earth. New York: Holt, primera edición.

(2001). Fire. Jeremy Mills Publishing.

Rambo, A. T. (1982). "Human Ecology Research on Tropical Agroecosystems in Southeast Asia”. Singapur Journal of Tropical Geography 3 (1): 3899.

Roebroeks, W. y P. Villa (2011). "On the Earliest Evidence for Habitual Use of Fire in Europe". Proceedings of the National Academy of Sciences 108 (13): 5209.

Smith, K. R. y J. L. Peel (2010). "Mind the Gap". Environmental Health Perspectives 118 (12): 1643-1645. 
Unger, N, T. C. Bond, J. S. Wang, D. M. Koch, S. Menon, D. T. Shindell y otros (2010). "Attribution of Climate Forcing to Economic Sectors". Proceedings of the National Academy of Sciences 107 (8): 3382-3387.

World Resource Institute (2007). Earth Trends. Environmental Information. Disponible en http://earthtrends.wri.org. 